\title{
In the eye of the beholder-rating of facial attractiveness in adult asperger syndrome
}

Mandy Roy ${ }^{1 *}$, Wolfgang Dillo and Lasse Osterhagen²

*Correspondence: Roy.Mandy@mh-hannover.de

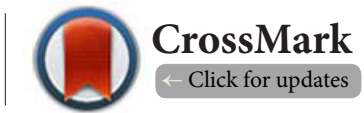

'Department of Psychiatry, Social Psychiatry and Psychotherapy, Hannover Medical School, Hannover, Germany. ${ }^{2}$ Department of Neuroscience, University of Oldenburg, Oldenburg, Germany.

\begin{abstract}
Background: Sense of facial attractiveness seems to be uniform within a particular culture and between different cultures in non-autistic persons. Individuals with an autism spectrum disorder have a different kind of perception and face-recognition compared to non-autistic persons, like a more detail-focused processing style and they show reduced activity in the face-specific gyrus fusiformis. Therefore it was aim of this study to find out if adults with an Asperger syndrome (AS) have difficulties in rating of facial attractiveness in comparison to non-autistic individuals.

Subjects and methods: 30 adults with an AS and 30 healthy controls (50\% female, range of age: 18-64 years) were instructed to judge attractiveness of 36 photographs of human faces with neutral expression. According to the hypothesis that a lower sense of facial attractiveness in AS would cause a stronger randomization in their rating, data were analyzed by comparing rating variances per face of both groups and conducting a random permutation test with analyzing the p-value and the Clopper-Pearson-CI.

Results: Random permutation test demonstrated stronger randomization of ratings in the AS group than in the control group $(\mathrm{p}=0.0045)$. Therefore it was concluded that individuals with AS have a lower sense of facial attractiveness. However, mean attractiveness ratings per face in the AS group was highly correlated with the mean attractiveness ratings in the control group $(\mathrm{r}=0.9447)$.

Conclusions: Results can be considered as reflecting uncertainties in attractiveness rating in AS, indicating a lower sense of facial attractiveness. Potentially contributing factors are discussed.
\end{abstract}

Keywords: Asperger syndrome, autism, perception, facial attractiveness

\section{Introduction}

Faces are complex visual stimuli that are based on a specific configuration of their attributes. Although variations in characteristics and their configuration are relatively small, each face expresses a different degree of attractiveness for most humans.

Several studies revealed an agreement about the sense of facial attractiveness within a particular culture as well as between different cultures [1]. Thus, facial attractiveness seems to be universal [2].

Factors that contribute to high facial attractiveness are a high degree of symmetry [3,4], averageness [5-7] as well as more feminine features in female faces $[8,9]$, and both male and feminine shapes in male faces [9]. Traits such as age, weight and hair seem to contribute to sensed attractiveness, too $[10,11]$. Faces that are showing a smile are rated as more attractive [12].
In addition to these factors, sensed facial attractiveness is affected by characteristics of the beholder. Some studies have shown an effect of hormone levels. Around ovulation women prefer masculine over feminine features in male faces [13], whereas men with higher testosterone levels prefer more female faces of women [14]. Personality traits of the beholder seem to influence the sense of attractiveness, too, as men with stronger tendencies to systemize-a sign of masculine personality trait-find more feminine features in women's faces more attractive. Women with a higher empathy, which is considered as a feminine personality trait, favor more masculine faces of men. These effects were not observed in the judgment of faces of the same sex [15]. Furthermore, previous exposure to certain facial traits seems to increase their attractiveness ratings [16]. Cerebral regions that are involved in the assessment of facial 
Roy et al. Journal of Autism 2016,

attractiveness are the left fronto-temporal cortex [17] and the medial orbitofrontal cortex [18].

All described studies have an important commonality: they refer to persons with a "normal kind" of perception and facerecognition. Thus it is unclear, if sense for facial attractiveness may be influenced by a "different way of perception". It is well known that autistic persons have a different kind of perception and face-recognition. Prevalence of autism spectrum disorders is estimated with $2.6 \%$ [19]. Besides problems in social interaction and stereotypic behavior, autistic individuals often show a stronger detail-focusing perception [20]. Happé and Frith postulated a "weak central coherence" referring to detail-focused processing style [20] with a stronger perception of local information instead of integrating details to a global form. But a more global context can be perceived if autistic individuals are instructed to. This predominance of local perception could influence the perception of faces and the sensed attractiveness. It is known that most types of face perception, such as recognition of identity, gender, or facial expression are based on a holistic perception in healthy persons [21]. Holistic perception means that the face is processed in its entirety, including the specific configuration of its features, in contrast to feature-based recognition. Abbas and Duchaine have shown that facial attractiveness is judged by a holistic processing of faces, too [21].

Autistic persons seem to process faces in a different way. They show difficulties in recognizing mimic expressions intuitively [22-24] especially regarding recognizing emotions from the eyes [25]. They often attend the mouth in contrast to the predominantly attention to the eyes of non-autistic persons [26]. There is also evidence for neurobiological abnormalities concerning face perception in autism. The fusiform gyrus is essential for cerebral processing of faces in humans [27] and it is also involved in the judgment of facial attractiveness [28]. In autistic persons, the neuronal activity in the fusiform gyrus as well as the connection between the fusiform gyrus and the amygdala is reduced while recognizing faces [29].

As visual perception and cerebral processing of faces seem to be altered in individuals with an autism spectrum disorder, it is unclear, if their sense of facial attractiveness is different in comparison to non-autistic individuals.

There is evidence that patients with acquired prosopagnosia, a deficit in recognizing familiar faces based on damage of medial occipitotemporal cortex, show deficits in the judgment of facial attractiveness. There by the severity of facial recognition impairment is positively correlated with difficulties in attractiveness rating [28].

It was aim of this study to test the hypothesis whether adults with an Asperger syndrome (AS), an autism spectrum disorder without delays in cognitive and lingual development, show difficulties in judging facial attractiveness in comparison to non-autistic persons.

Thus, an insight into the literally "world outlook" of autistic persons should be gained for improving the understanding of perception in autism.

\section{Methods \\ Subjects}

We included 30 adults with the diagnosis of AS (15 female, 15 male adults, range of age: $21-58$ years, mean: 42.5 years) and 30 healthy controls ( 15 female, 15 male adults, range of age: 18-64 years, mean: 36.9 years). Adults with AS were recruited from our outpatient clinic for AS. Healthy controls were recruited from the staff of our clinic, which was not otherwise involved in the research project.

\section{Assessment}

AS in adulthood was diagnosed using a self-developed, semi-structured interview (Diagnostic interview: Asperger syndrome in adulthood) [30] that thoroughly assessed the patients according to DSM-IV criteria. After a general section focusing on medical anamnesis (somatic, psychiatric, and social histories, including childhood development), the interview continues with a special section involving AS that includes the following items with respect to childhood and adulthood: social interaction and communication (e.g., friendships with/relationship to/interest in peers, and being a loner and suffering from loneliness); special interests (e.g., spending leisure time, and interest in specific objects/topics); stereotypic behavior (e.g., rituals, and reaction towards disturbances of rituals); and other characteristics (e.g., clumsiness, and sensitivity towards noises/smells/tactile stimuli). Additionally, eye contact, mimicking expressions, speech melody, "mirroring" of affections, and clumsiness were observed during the interview. The interview was conducted by the same experienced investigator and had a duration of approximately 90 minutes. If available, diagnosis of AS was complemented by information from personal/telephone interviews, or in written form from observers during childhood and/or adulthood, such as partners, friends, parents, or siblings. In some cases, school reports were consulted. The diagnosis of AS was only confirmed if DSM-IV criteria were clearly fulfilled based on clinical judgment and available information during the interview.

There is no standardized interview or test available for diagnosing AS in adults according to DSM-IV/ DSM- 5 criteria that is based on information obtained from sources other than parents and adults often do not wish parents to be consulted.

Additionally, we used the two self-rating scales "Autismspectrum quotient" (AQ) [31] and "Empathy quotient" (EQ) [32]. The $A Q$ is a scale for quantification, in which the score of a person is assessed on a continuum from normality to autism; a higher score indicates more pronounced autistic traits. The EQ is a scale for estimating an individual's ability to empathize, thereby a higher score indicates stronger empathy. Baron-Cohen suggested a cut-off of $\geq 32$ points for the AQ and $\leq 30$ points for the $E Q$.

In our sample of adults with AS only two female persons 
scored 31 respectively 27 points in the $A Q$, but 27 respectively 21 points in the $E Q$, thus we did not exclude them. All other adults with $A S$ scored above the cut-off of the $A Q$ and below the cut-off of the EQ.

Healthy controls had no diagnosis of an autistic spectrum disorder and no other current psychiatric disorders. All controls scored below the cut-off of the AQ and above the cut-off of the $E Q$, there was no indication for an autism spectrum disorder.

No autistic or non-autistic participant had a mental retardation, as tested by a German multiple-choice word recognition test for the measurement of intelligence ("MehrfachwahlWortschatz-Intelligenztest MWT-B", Lehrl 1993).

All participants gave informed consent after the procedure had been explained. Approval for this study was given by the Ethics Committee of Hannover Medical School.

\section{Stimuli and presentation}

We used 36 coloured photographs of human faces ( 18 female, 18 male faces) with neutral facial expressions. Photographs were taken with permission from the FACES database" of the Max-Planck Institute for Human Development, Berlin, Germany [33] (Figure 1), for technical details of the photographs, see reference. Photos of the size $9.8 \times 12.3 \mathrm{~cm}$ were presented on a $56 \times 35 \mathrm{~cm}$ screen. All participants could freely choose their distance to the screen. For all participants, the stimuli were presented in the same randomized order. Presentation of the photographs started with a first run presenting each picture for $1500 \mathrm{~ms}$ for giving the participants a first impression of the stimuli, thus they could efficiently make use of the rating scale's range in a second run. In the second run the attractiveness of the faces should be judged on a scale ranging from 1 (labeled "not attractive at all") to 10 (labeled "extremely attractive"). Time was recorded but there were no time constrictions; to continue to the next photograph, a rating must have been given.

\section{Data analysis}

Data were analyzed in the following way: If AS individuals have

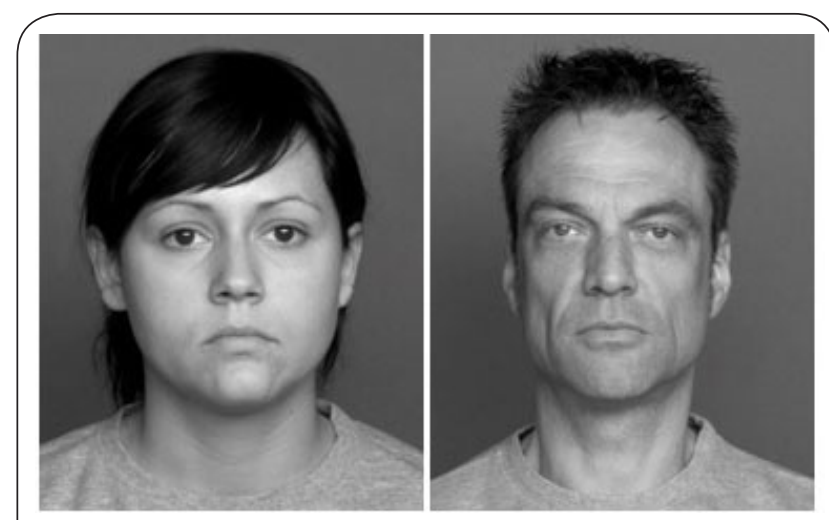

Figure 1. Photographs of human faces with a neutral facial expression. Photos were shown in colors and should be rated on a scale from 1 (not attractive at all) to 10 (extremely attractive). a lower sense of facial attractiveness than control subjects, their ratings will be somewhat more random than those of the controls. This will be reflected in higher rating variances per face within the AS group in comparison to the rating variances per face within the control group, significance of difference in variances was then conducted by a random permutation test, the difference between the sums of variances served as our observed test statistic [34,35].

According to this procedure we computed the sum of all 36 rating variances per face in the AS group and did the same in the control group. We then generated new groups by randomly assigning 30 participants to one group and the remaining participants to a second group. We then again computed the difference between the sum of variances in the first and the second group and compared it to our observed test statistic. We repeated the procedure one million times and analyzed the $\mathrm{p}$-value and the Clopper-Pearson-CI [36].

Furthermore we conducted the correlation of mean attractiveness ratings per face between both groups and the correlation between deviations from average attractiveness ratings and $A Q$ and $E Q$ respectively [37]. Total rating time between both groups was compared via t-Test.

\section{Results}

In Table 1 means and variances of attractiveness ratings are listed. Figure 2 demonstrates boxplots of ratings in our studygroup. Results show a similar pattern of rating between both study groups with greater ranges of rating in the AS group.

Data analysis revealed a higher sum of variances in the AS group (152.7977) than in the control group (84.0839). After proceeding with generating new random groups and computing differences between sum of variances one million
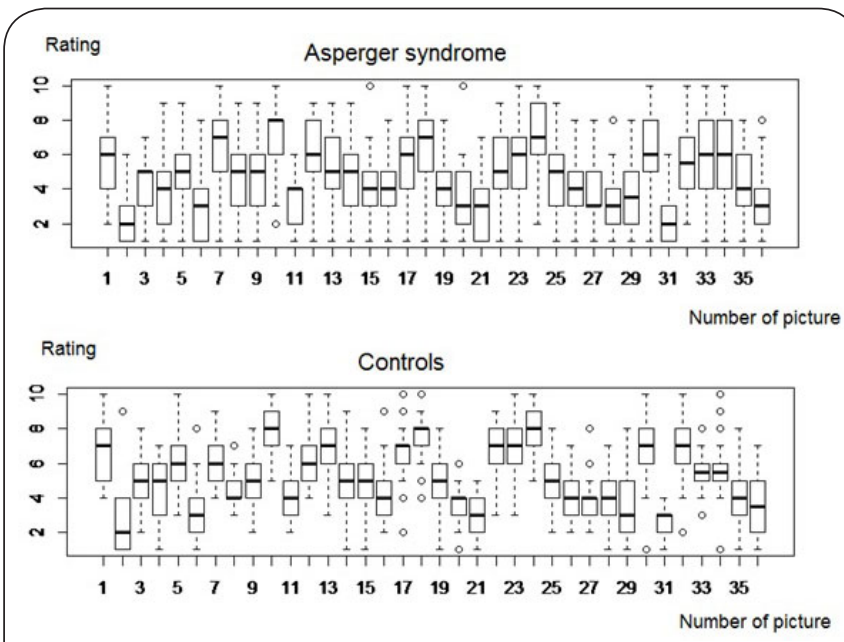

Figure 2. Boxplots of facial attractiveness rating in AS and controls. Displayed are the interquartil ranges (boxes), the complete ranges of rating (dashed line) and the medians (bold line). Correlation of both study groups is high, but randomization in AS is significantly higher. 
Roy et al. Journal of Autism 2016,

Table 1. Means and variances of attractiveness ratings of AS and control group per photo.

\begin{tabular}{|c|c|c|c|c|}
\hline \multirow{2}{*}{$\begin{array}{l}\text { Number } \\
\text { of photo }\end{array}$} & \multicolumn{2}{|c|}{ Asperger syndrome } & \multicolumn{2}{|c|}{ Controls } \\
\hline & Mean & Variance & Mean & Variance \\
\hline 1 & 5.60 & 4.662 & 6.57 & 2.737 \\
\hline 2 & 2.50 & 2.190 & 2.60 & 2.800 \\
\hline 3 & 4.10 & 2.783 & 4.73 & 2.823 \\
\hline 4 & 3.73 & 3.306 & 4.27 & 3.237 \\
\hline 5 & 5.03 & 4.654 & 6.03 & 2.999 \\
\hline 6 & 3.20 & 4.028 & 3.03 & 2.516 \\
\hline 7 & 6.47 & 5.361 & 5.90 & 1.817 \\
\hline 8 & 4.93 & 4.478 & 4.47 & 1.154 \\
\hline 9 & 4.60 & 4.248 & 5.07 & 1.789 \\
\hline 10 & 7.23 & 3.978 & 8.10 & 1.266 \\
\hline 11 & 3.43 & 2.392 & 4.13 & 1.637 \\
\hline 12 & 6.07 & 4.478 & 6.33 & 2.299 \\
\hline 13 & 5.30 & 3.459 & 6.63 & 2.516 \\
\hline 14 & 4.60 & 4.593 & 5.17 & 2.764 \\
\hline 15 & 4.20 & 5.131 & 4.97 & 3.137 \\
\hline 16 & 3.93 & 2.892 & 4.30 & 2.355 \\
\hline 17 & 5.57 & 5.082 & 6.73 & 2.754 \\
\hline 18 & 6.30 & 6.148 & 7.57 & 1.771 \\
\hline 19 & 4.10 & 4.300 & 4.63 & 3.275 \\
\hline 20 & 3.50 & 3.983 & 3.67 & 1.264 \\
\hline 21 & 2.87 & 2.809 & 3.07 & 1.375 \\
\hline 22 & 5.07 & 4.409 & 6.53 & 2.947 \\
\hline 23 & 5.87 & 5.085 & 6.90 & 2.714 \\
\hline 24 & 6.93 & 4.409 & 7.97 & 1.689 \\
\hline 25 & 4.70 & 4.700 & 5.03 & 2.999 \\
\hline 26 & 3.97 & 3.620 & 3.90 & 1.541 \\
\hline 27 & 3.67 & 3.885 & 3.73 & 1.720 \\
\hline 28 & 3.43 & 3.082 & 3.90 & 2.783 \\
\hline 29 & 3.57 & 4.047 & 3.50 & 2.672 \\
\hline 30 & 5.97 & 6.171 & 6.53 & 3.637 \\
\hline 31 & 2.47 & 2.051 & 2.50 & 1.017 \\
\hline 32 & 5.73 & 5.099 & 6.70 & 2.493 \\
\hline 33 & 5.83 & 6.213 & 5.50 & 1.914 \\
\hline 34 & 5.80 & 6.234 & 5.67 & 2.989 \\
\hline 35 & 4.37 & 4.447 & 4.10 & 2.093 \\
\hline 36 & 3.43 & 4.392 & 3.60 & 2.593 \\
\hline
\end{tabular}

times, we only found in 4495 cases test statistics that were higher than the observed test statistic. This gave a significant $p$-value of $p=0.0045$ with a Clopper-Pearson-Cl between 0.0044 and 0.0046 , indicating that ratings of the AS group were more random than those of the controls. Therefore, we may conclude that AS individuals have a lower sense of facial attractiveness than non-AS controls.

We found the mean attractiveness ratings per face in the AS group to be highly correlated with the mean attractiveness ratings in the control group ( $r=0.9447)$.

AS' uncertainties in attractiveness ratings could be canceled through the process of averaging, which would be an example of the wisdom of crowds [38]. To further verify this conclusion, we computed the overall mean of the attractiveness ratings per face and for each participant the absolute deviations of his ratings from the overall means. The higher the sum of these deviations, the less are the participant's attractiveness ratings in accordance with the common perception of attractiveness. We expected the ratings of AS individuals to deviate more from the average attractiveness ratings than those of the controls. This hypothesis was confirmed by a Wilcoxon rank-sum test $(\mathrm{W}=328$, one-sided $p=0.0362$ ). Furthermore, ratings of people with higher autistic traits, measured by the $A Q$, deviate more from the common view of facial attractiveness than those of people with lower autistic traits.

Deviations from average attractiveness ratings were positively correlated with the $A Q(r=0.2195)$ and negatively correlated with the EQ ( $r=-0.1855)$, but only the former being significant (one-tailed $p=0.0460$ ). Nevertheless, correlations can be only regarded as weak.

There was no significant difference regarding the total rating time between both groups (mean time AS group $=254671$ msec, mean time controls $=257073 \mathrm{msec}, \mathrm{p}=0.96$ ).

\section{Discussion}

It was aim of this study to find out whether persons with an autism spectrum disorder such as AS, have a different sense of facial attractiveness.

We found the attractiveness rating agreement to be significantly lower within the AS group in comparison to the non-autistic controls. Because a low agreement means a higher degree of randomness in the ratings, we may consider this as a result of guessing, reflecting autists' uncertainties with regard to the attractiveness rating of these stimuli. This indicates a lower sense of facial attractiveness in our AS study group rather than a systematic different but homogenous sense within this group. This assumption is supported by the fact that their average ratings highly correlate with those of the control subjects, what contradicts a systematic different sense of facial attractiveness. But this does not necessarily refute the above-mentioned conclusion. Furthermore there was no significant difference in the total rating time between both groups. On the one hand uncertainties could induce a longer decision-time, on the other hand a kind of guessing could cause a faster decision. It remains unclear if both aspects counterbalance each other and lead to a similar rating time or if a lower sense of facial attractiveness does not affect the reaction time in a significant extent.

There are several factors that may contribute to our findings. As described above, symmetry is an important factor for facial attractiveness $[3,4]$. According to a common assumption, autistic persons are considered as sensitive towards symmetry. Therefore, intuitively one could reason, that they have a fine sense for facial attractiveness based on symmetry. However, Falter hypothesized that autistics' recognition of symmetry 
is relating to a local level, but is impaired if it requires global attention [39]. This is in accordance with the findings that AS individuals prefer a detail-focused processing style [20]. As in non-autistic persons sense of facial attractiveness is based on a holistic style of processing [21], it could be concluded that individuals with AS may have difficulties to use holistic facial symmetry as a factor for rating facial attractiveness.

Another factor for facial attractiveness is averageness $[5,6]$. Rhodes et al. showed that sensed averageness thereby strongly depends on preceding experiences with faces [40]. For example, a short exposure to distorted faces is able to change the sensed normality of presented faces towards the distortion in non-autistic individuals. As autistic persons show a lack of interest in faces [41-43], their experiences regarding faces may be reduced. Thus, the low experience with faces may lead to a reduced sense of facial averageness in autists and consecutively to the lower sense of facial attractiveness.

Additionally, Rhodes et al. described averageness as a "central location" in a distribution of faces rather than an "intrinsic appeal of particular physical characteristics" [40]. According to this hypothesis, facial averageness seems to be represented in the whole facial configuration, requiring a holistic perception. Here again, the lower sense of facial attractiveness could be a result of the subordination of holistic visual perception in autistic persons.

For most non-autistic persons, faces are of great importance because of their social function, such as presenting emotions and intentions. Therefore, in interaction they focus on the face. Autistic individuals often avoid looking at the others' face $[44,45]$ and have difficulties with intuitively recognizing mimics [22-25]. It could be concluded that the human face doesn't have the importance for autistic persons as it has for non-autistic individuals. It could be hypothesized that it is easier to develop aesthetic values for stimuli of great importance and vice versa to not develop aesthetic values for stimuli of less personal importance. For example, one might assume that non-autistic individuals may have difficulties in judging attractiveness of stimuli with a low impact for them, too, e.g., for faces of cattle (at least if he is not a cattle breeder).

Yet it is unclear how to interpret the reduced neuronal activity in face-relevant cerebral areas in autistic persons, especially in the fusiform gyrus (FG) [29]. It is still a controversial discussion, if a special area in the fusiform gyrus, called 'fusiform face area' (FFA), reacts selectively and exclusively towards faces or if it is reacting towards stimuli, the individual person has got a lot of experience with. There is evidence for this latter hypothesis as a study investigated children with great expertise concerning Pokémon cards and control children that do not [46]. Expert children showed increased activity in FFA during viewing Pokémon characters in contrast to control children. Nevertheless, the 'face selective view' and the 'expert hypothesis' are not mutually exclusive, as, because of the importance of faces during interaction, we can consider non-autistic humans to be experts for faces. Autistic persons are not. Maybe their reduced looking at faces don't let them become experts for faces with a consecutively reduced activity in FG. Vice versa, their problems in decoding mimic information of faces may be a result from the dysfunction of the FG.

It is also unclear, whether reduced activity in the FG is a result of its own dysfunction or a result of reduced bottom-up information that is entering the FG. The "fast-track modulator model" suggests impairment in the subcortical face detecting route including amygdala, pulvinar and superior colliculus with a modulated subcortical-cortical connectivity in autism spectrum disorders [47]. Especially, the functional connectivity between amygdala and fusiform gyrus seems to be diminished in autism [48]. However, dysfunction of the fusiform gyrus in autism will probably contribute to the low sense of facial attractiveness, as it is known to be involved in the process of judging attractiveness [28].

Our findings may also contribute to the explanation of further phenomena. It is well known that in non-autistic individuals facial attractiveness influences judgments about others concerning desirability, personality or competency in work $[49,50]$. Autistic persons show deficits in deriving social judgements from human faces [51], what could be associated with the lower sense of attractiveness. Mesibov and Stephens investigated 16 high-functioning autistic adults who were members of a social skills group for several years [52]. They should evaluate the other group members inter alia for attractiveness and popularity. A high correlation between perceived attractiveness and popularity was found, but their perception of attractiveness was quite different from those of healthy group leaders, who served as controls. Mesibov and Stephens concluded, that for autistic persons, attractiveness is an important factor for popularity, just as it is for non-autists. With regard to our findings, the reversed process could also be possible, the autistic adults could have based their assessment of attractiveness on their sensed popularity towards the rated persons.

Further investigation could address modulation of attractiveness ratings in autism by emotional expressions, as it is known that non-autistic persons rate smiling faces as more attractive [12] and if facial attractiveness impacts partner-choice and motivates sexual behavior, like in non-autistic persons [53]. Finally, it could be object of interest, as faces don't seem to have this aesthetic meaning for autistic individuals like they have for non-autists, which kind of stimuli do have for autists.

\section{Limitations of the study}

One limitation of our study is the small number of participants. Another general problem is the lack of a gold standard in the diagnosis of adult AS. Various interviews are not consistent with DSM-IV criteria or are based on information obtained from parents concerning the childhood of the individuals. But often parents are not available or adults do not wish to involve them. According to Joshi et al [54], we gave priority to consideration of the DSM-IV criteria by interview and clinical 
Roy et al. Journal of Autism 2016,

observation. Thus, we can clearly state that all included adults fulfilled the DSM-IV criteria for AS.

Results showed a relatively small range of attractiveness rating in the control-group of non-autistic individuals, only few photographs were rated as particular attractive or nonattractive. Results between individuals with AS and non-autistic persons could have been different, if differences between photographs would have been more pronounced. On the other hand, differences between both groups that are based on photos without a great range in attractiveness seem to be more meaningful, indicating subtle differences in the sense for attractiveness between both study groups.

\section{Conclusions}

Individuals with AS seem to have rather a lower than a systematic different sense of facial attractiveness. This may be a result of the aberrant kind of perception in autism and may contribute to the altered importance of faces in contrast to non-autistic persons. As faces don't appear to be a prominent target for sensed attractiveness in AS it would be an issue of interest, what kind of visual stimuli are.

\section{Competing interests}

The authors declare that they have no competing interests.

Authors' contributions

\begin{tabular}{|l|c|c|c|}
\hline Authors' contributions & MR & WD & LO \\
\hline Research concept and design & $\checkmark$ & $\checkmark$ & $\checkmark$ \\
\hline Collection and/or assembly of data & $\checkmark$ & $\checkmark$ & $\checkmark$ \\
\hline Data analysis and interpretation & $\checkmark$ & -- & $\checkmark$ \\
\hline Writing the article & $\checkmark$ & $\checkmark$ & $\checkmark$ \\
\hline Critical revision of the article & $\checkmark$ & $\checkmark$ & $\checkmark$ \\
\hline Final approval of article & $\checkmark$ & $\checkmark$ & $\checkmark$ \\
\hline Statistical analysis & -- & -- & $\checkmark$ \\
\hline
\end{tabular}

Publication history

Editor: David Reiss, Imperial College London, UK. Received: 27-Mar-2016 Final Revised: 07-Apr-2016

Accepted: 19-Apr-2016 Published: 28-Apr-2016

\section{References}

1. Langlois JH, Kalakanis L, Rubenstein AJ, Larson A, Hallam M and Smoot M. Maxims or myths of beauty? A meta-analytic and theoretical review. Psychol Bull. 2000; 126:390-423. | Article | PubMed

2. Little $A C$, Jones $B C$ and DeBruine $L M$. Facial attractiveness: evolutionary based research. Philos Trans R Soc Lond B Biol Sci. 2011; 366:1638-59. | Article | PubMed Abstract | PubMed FullText

3. Grammer $K$ and Thornhill R. Human (Homo sapiens) facial attractiveness and sexual selection: the role of symmetry and averageness. J Comp Psychol. 1994; 108:233-42. | Article | PubMed

4. Scheib JE, Gangestad SW and Thornhill R. Facial attractiveness, symmetry and cues of good genes. Proc Biol Sci. 1999; 266:1913-7. I Article | PubMed Abstract | PubMed FullText

5. Langlois JH and Roggman LA. Attractive Faces Are Only Average. Psychological Science. 1990; 1:115-121. | Article

6. Rhodes $\mathrm{G}$ and Tremewan T. Averageness, Exaggeration, and Facial
Attractiveness. Psychological Science. 1996; 7:105-110. | Article

7. Jones $D$ and Hill K. Criteria of facial attractiveness in five populations. Hum Nat. 1993; 4:271-96. | Article | PubMed

8. Cunningham MR. Measuring the physical in physical attractiveness: Quasi-experiments on the sociobiology of female facial beauty. Journal of Personality and Social Psychology. 1986; 50:925-935.

9. Perrett DI, Lee KJ, Penton-Voak I, Rowland D, Yoshikawa S, Burt DM, Henzi SP, Castles DL and Akamatsu S. Effects of sexual dimorphism on facial attractiveness. Nature. 1998; 394:884-7. | Article | PubMed

10. Korthase KM and Trenholme I. Perceived age and perceived physical attractiveness. Perceptual and Motor Skills. 1982; 54:1251-1258. | Article

11. Coetzee V, Perrett DI and Stephen ID. Facial adiposity: a cue to health? Perception. 2009; 38:1700-11. | Article | PubMed

12. Otta E, Folladore Abrosio F and Hoshino RL. Reading a smiling face: messages conveyed by various forms of smiling. Percept Mot Skills. 1996; 82:1111-21. | Article | PubMed

13. Penton-Voak IS, Perrett DI, Castles DL, Kobayashi T, Burt DM, Murray LK and Minamisawa R. Menstrual cycle alters face preference. Nature. 1999; 399:741-2. | Article | PubMed

14. Welling LL, Jones BC, DeBruine LM, Smith FG, Feinberg DR, Little AC and Al-Dujaili EA. Men report stronger attraction to femininity in women's faces when their testosterone levels are high. Horm Behav. 2008; 54:703-8. | Article | PubMed

15. Finlay G. Smith, Benedict C. Jones and Lisa M. DeBruine. Individual differences in empathizing and systemizing predict variation in face preferences. Personality and Individual Differences. 2010; 49:655-658. | Article

16. Gavin Buckingham, Lisa M. DeBruine, Anthony C. Little, Lisa L.M. Welling, Claire A. Conway, Bernard P. Tiddeman and Benedict C. Jones. Visual adaptation to masculine and feminine faces influences generalized preferences and perceptions of trustworthiness. Evolution \& Human Behavior. 2006; 27:381-389. | Article

17. Nakamura K, Kawashima R, Nagumo S, Ito K, Sugiura M, Kato T, Nakamura A, Hatano K, Kubota K, Fukuda H and Kojima S. Neuroanatomical correlates of the assessment of facial attractiveness. Neuroreport. 1998; 9:753-7. | Article | PubMed

18. O'Doherty J, Winston J, Critchley H, Perrett D, Burt DM and Dolan RJ. Beauty in a smile: the role of medial orbitofrontal cortex in facial attractiveness. Neuropsychologia. 2003; 41:147-55. I Article I PubMed

19. Kim YS, Leventhal BL, Koh YJ, Fombonne E, Laska E, Lim EC, Cheon KA, Kim SJ, Kim YK, Lee H, Song DH and Grinker RR. Prevalence of autism spectrum disorders in a total population sample. Am J Psychiatry. 2011; 168:904-12. | Article | PubMed

20. Happe $F$ and Frith $U$. The weak coherence account: detail-focused cognitive style in autism spectrum disorders. J Autism Dev Disord. 2006; 36:5-25. | Article | PubMed

21. Abbas ZA and Duchaine $B$. The role of holistic processing in judgments of facial attractiveness. Perception. 2008; 37:1187-96. | Article | PubMed

22. Hobson RP. The autistic child's appraisal of expressions of emotion. $J$ Child Psychol Psychiatry. 1986; 27:321-42. I Article | PubMed

23. Tantam D, Monaghan L, Nicholson $\mathrm{H}$ and Stirling J. Autistic children's ability to interpret faces: a research note. J Child Psychol Psychiatry. 1989; 30:623-30. | Article | PubMed

24. Hobson RP, Ouston J and Lee A. What's in a face? The case of autism. $\mathrm{Br}$ J Psychol. 1988; 79 ( Pt 4):441-53. | Article | PubMed

25. Baron-Cohen S, Jolliffe T, Mortimore C and Robertson M. Another advanced test of theory of mind: evidence from very high functioning adults with autism or asperger syndrome. J Child Psychol Psychiatry. 1997; 38:813-22. | Article | PubMed

26. Klin A, Jones W, Schultz R, Volkmar F and Cohen D. Visual fixation patterns during viewing of naturalistic social situations as predictors of social competence in individuals with autism. Arch Gen Psychiatry. 2002; 59:809-16. | Article | PubMed

27. Kanwisher N, McDermott J and Chun MM. The fusiform face area: a module in human extrastriate cortex specialized for face perception. $J$ 
Roy et al. Journal of Autism 2016,

Neurosci. 1997; 17:4302-11. | Article | PubMed

28. Iaria G, Fox CJ, Waite CT, Aharon I and Barton JJ. The contribution of the fusiform gyrus and superior temporal sulcus in processing facial attractiveness: neuropsychological and neuroimaging evidence. Neuroscience. 2008; 155:409-22. | Article | PubMed Abstract | PubMed FullText

29. Dziobek I, Bahnemann M, Convit A and Heekeren HR. The role of the fusiform-amygdala system in the pathophysiology of autism. Arch Gen Psychiatry. 2010; 67:397-405. | Article | PubMed

30. Roy M, Prox-Vagedes V, Ohlmeier MD and Dillo W. Beyond childhood: psychiatric comorbidities and social background of adults with Asperger syndrome. Psychiatr Danub. 2015; 27:50-9. | Pdf | PubMed

31. Baron-Cohen S, Wheelwright S, Skinner R, Martin J and Clubley $\mathrm{E}$. The autism-spectrum quotient (AQ): evidence from Asperger syndrome/high-functioning autism, males and females, scientists and mathematicians. J Autism Dev Disord. 2001; 31:5-17. | Article | PubMed

32. Baron-Cohen $S$ and Wheelwright $S$. The empathy quotient: an investigation of adults with Asperger syndrome or high functioning autism, and normal sex differences. J Autism Dev Disord. 2004; 34:16375. | Article | PubMed

33. Ebner NC, Riediger M and Lindenberger U. FACES--a database of facial expressions in young, middle-aged, and older women and men: development and validation. Behav Res Methods. 2010; 42:351-62. I Article | PubMed

34. Fisher RA. The Design of Experiments. Hafner, New York. 1935.

35. Good PI. Permutation, Parametric and Bootstrap Tests of Hypotheses, 3rd ed. Springer. 2005.

36. Krengel U. Einführung in die Wahrscheinlichkeitstheorie und Statistik. 8. Auflage. Vieweg. 2005.

37. Bühner $M$ and Ziegler M. Statistik für Psychologen und Sozialwissenschaftler. Pearson Studium. 2009.

38. Galton F. Vox populi. Nature. 1949; 450-451.

39. Falter CM. Impaired and superior mirror symmetry perception in autism. Autism. 2013; 17:117-8. | Article | PubMed

40. Rhodes G, Jeffery L, Watson TL, Clifford CW and Nakayama K. Fitting the mind to the world: face adaptation and attractiveness aftereffects. Psychol Sci. 2003; 14:558-66. | Article | PubMed

41. Riby DM and Hancock PJ. Viewing it differently: social scene perception in Williams syndrome and autism. Neuropsychologia. 2008; 46:2855-60. | Article | PubMed

42. Riby DM and Hancock PJ. Do faces capture the attention of individuals with Williams syndrome or autism? Evidence from tracking eye movements. J Autism Dev Disord. 2009; 39:421-31. | Article | PubMed

43. Riby DM, Brown PH, Jones $\mathrm{N}$ and Hanley M. Brief report: faces cause less distraction in autism. J Autism Dev Disord. 2012; 42:634-9. | Article I PubMed

44. Adrien JL, Faure M, Perrot A, Hameury L, Garreau B, Barthelemy C and Sauvage D. Autism and family home movies: preliminary findings. $J$ Autism Dev Disord. 1991; 21:43-9. | Article | PubMed

45. Hobson RP and Lee A. Hello and goodbye: a study of social engagement in autism. J Autism Dev Disord. 1998; 28:117-27. | Article | PubMed

46. James TW and James KH. Expert individuation of objects increases activation in the fusiform face area of children. Neuroimage. 2013; 67:182-92. | Article | PubMed

47. Senju A and Johnson MH. Atypical eye contact in autism: models, mechanisms and development. Neurosci Biobehav Rev. 2009; 33:120414. | Article | PubMed

48. Kleinhans NM, Richards T, Sterling L, Stegbauer KC, Mahurin R, Johnson LC, Greenson J, Dawson G and Aylward E. Abnormal functional connectivity in autism spectrum disorders during face processing. Brain. 2008; 131:1000-12. | Article | PubMed

49. Dion K, Berscheid E and Walster E. What is beautiful is good. J Pers Soc Psychol. 1972; 24:285-290. | Article

50. Dipboye RL, Formkin HL and Wiback K. Relative importance of applicant sex, attractiveness, and scholastic standing in evaluation of job applicant resumes. Journal of Applied Psychology. 1975; 60:39-43.

51. Philip RC, Whalley HC, Stanfield AC, Sprengelmeyer R, Santos IM, Young AW, Atkinson AP, Calder AJ, Johnstone EC, Lawrie SM and Hall J. Deficits in facial, body movement and vocal emotional processing in autism spectrum disorders. Psychol Med. 2010; 40:1919-29. | Article | PubMed

52. Mesibov GB and Stephens J. Perceptions of popularity among a group of high-functioning adults with autism. J Autism Dev Disord. 1990; 20:3343. | Article | PubMed

53. Rhodes $G$. The evolutionary psychology of facial beauty. Annu Rev Psychol. 2006; 57:199-226. | PubMed

54. Joshi G, Wozniak J, Petty C, Martelon MK, Fried R, Bolfek A, Kotte A, Stevens J, Furtak SL, Bourgeois M, Caruso J, Caron A and Biederman J. Psychiatric comorbidity and functioning in a clinically referred population of adults with autism spectrum disorders: a comparative study. J Autism Dev Disord. 2013; 43:1314-25. | Article | PubMed

\section{Citation:}

Roy M, Dillo W and Osterhagen L. In the eye of the beholder-rating of facial attractiveness in adult asperger syndrome. J Autism. 2016; 3:2.

http://dx.doi.org/10.7243/2054-992X-3-2 